\title{
EDITORIAL
}

\section{Release of neurotransmitters from glia}

\author{
R. DOUGLAS FIELDS
}

\begin{abstract}
There is no question about the fact that astrocytes and other glial cells release neurotransmitters that activate receptors on neurons, glia and vascular cells, and that calcium is an important second messenger regulating the release. This occurs in cell culture, tissue slice and in vivo. Negative results from informative experiments designed to test the mechanism of calciumdependent neurotransmitter release from astrocytes and the ensuing effects on synaptic transmission, have been cited as evidence calling into question whether astrocytes release neurotransmitters under normal circumstances with effects on synaptic transmission. The special feature section in this issue of Neuron Glia Biology addresses these issues and other aspects of neurotransmitter release from astrocytes in communicating with neurons and glial cells. Together these studies suggest that application of vocabulary and concepts developed for synaptic communication between neurons can lead to confusion and apparent paradoxes with respect to communication by extracellular signaling molecules released from glia in response to functional activity.
\end{abstract}

Keywords: neurotransmitter release, synaptic transmission, synaptic vesicle, LTP, astrocytes, tripartite synapse, neuron-glia interactions, calcium, intercellular signaling

Classically, neurotransmitters are understood to be small organic molecules packaged into synaptic vesicles of nerve terminals and released rapidly in response to calcium influx through voltage-gated calcium channels to activate receptors that depolarize or hyperpolarize the postsynaptic membrane of adjacent neurons. (An exception is recognized to include communication between motor neurons and muscle cells.) The discovery of 'neurotransmitter' release from glia raises the question of whether usage of this word should be expanded to include molecules released from other types of nervous system cells, and likely through different cellular mechanisms, for the purpose of intercellular communication in the nervous system.

Should the same molecule, glutamate, for example be called a neurotransmitter when it is released from axons through membrane channels, rather than from synaptic vesicles, or released by reversal of amino acid transporters in communicating with other neurons? Or, should glutamate, when released from neurons through non-vesicular mechanisms, be referred to by some different name. Likewise, when glutamate is released from an astrocyte, should it be called a 'gliotransmitter', even when this mediates communication with neurons? An answer to the evolving meaning of the term neurotransmitter might depend on whether the source, recipient or mode of transmission should be the defining element in discussing transmission of information in general, and appreciating that the root 'neuro' denotes nervous system, not neuron. The discovery of neurotransmitters (Dale, 1914; Loewi, 1921) preceded both the concept of the synaptic vesicle (Palade and Palay, 1954; De Robertis and Bennett, 1955), and all the mechanisms that have been worked out since for its calciumdependent release (Katz and Miledi, 1965). Indeed, both the concept and the mechanisms for synaptic vesicle release were first described not in communication between neurons, but rather in synaptic communication at junctions between neurons and non-neuronal cells (muscle).
Precision in language is critical, but language is not rigid. The danger of an inflexible terminology is that adherence to out-dated vocabulary can constrain thinking - just as the root 'glue' did for neuroglia. Language will necessarily evolve over time to give structure and meaning to description and to reflect new understanding. Mismatches between terms and mechanisms when applied in new contexts can reveal new aspects of biology (Agulhon et al., 2010), not necessarily that the observation stimulating the investigation is an artifact (Stern, 2010; Wiedemann, 2010).

A rise in cytoplasmic calcium is mechanistically linked to release of neurotransmitter from astrocytes, but how this occurs has been a mystery from the outset, as was noted in a general review on glia, published in 2002 (Fields and Stevens-Graham, 2002). After nearly a decade of research this problem remains, but significant progress has been made (Parpura and Haydon, 2009). Neurotransmitters are released from astrocytes by the membrane fusion of vesicles that resemble synaptic vesicles structurally and biochemically in neurons, and also from other types of vesicles, for example, those resembling lysosomes (Zhang et al., 2007). Neurotransmitters are also released from neurons and glia through channels of various types (Fields and $\mathrm{Ni}, 2010$ ) and by membrane transporters (Baltan, 2009), but glia are fundamentally different from neurons. The function, mechanisms, spatial and temporal aspects of communication and the diverse range of recipient nervous system cells participating in glial communication differ radically from neuronal communication. It should not be expected that calcium influx would regulate release of neurotransmitter from astrocytes in precisely the same way it does at synapses between neurons.

Two papers by Achour et al. (2010) and Henneberger and Rusakov (2010) in this issue provide comprehensive reviews of calcium signaling and neurotransmitter release from astrocytes, and the involvement of neurotransmitter release from astrocytes in long-term potentiation (LTP). These papers 
also consider apparent contradictions in studies that probe this question of astrocyte contribution to neural plasticity via calcium-dependent neurotransmitter release (Fiacco et al., 2007; Agulhon et al., 2010). Achour et al. conclude that the kinetics of calcium response are important in neurotransmitter release from astrocytes, and that slow calcium oscillation rather than large increases in calcium can be important, as well as differences between submembrane calcium transients versus bulk cytoplasmic calcium changes. Differences in glutamate and D-serine release in affecting synaptic plasticity and different stimulus patterns used to induce LTP can affect the concentration of glutamate in the extracellular space and thus intercellular signaling and synaptic plasticity. Henneberger and Rusakov show that standard calcium imaging does not reveal calcium-dependent signaling inside astrocytes with sufficient resolution to monitor changes in the nanodomains that are thought to be controlling neurotransmitter release, primarily because the relevant astrocyte cellular processes ensheathing synapses are too fine, by an order of magnitude, to resolve with standard fluorescence imaging. Both papers highlight the need for further research in investigating calcium signaling at astrocyte microdomains in fine process associated with synapses near the sites of glutamate release, and both argue that improved methods of calcium monitoring in situ will be essential to resolve the ambiguity in understanding the calcium signaling cascades regulating neurotransmitter release from glia.

In an original paper by Shigetomi et al. (2010) the researchers explore better methods of calcium measurement for these purposes. In these studies a genetically encoded calcium indicator has been modified with a membrane-tethering domain. This modification positions the sensor to better monitor the submembrane calcium transients, and the results reveal novel microdomains of calcium signals in astrocytes that are quite distinct from global changes in calcium. They conclude that the type of calcium channel, location and dynamics of calcium signals in astrocytes are likely to affect how neurotransmitter release is regulated by calcium ions.

Dave et al. (2010) show that astrocytes in the subventricular zone (SVZ) release glutamate upon intracellular calcium increases, and they seek to determine what controls the calcium transient and glutamate release. Using patch clamp recording of neuroblasts to measure $N$-methyl-D-aspartate (NMDA) receptor currents as a monitor of extracellular glutamate levels, their research indicates that that adding prostaglandin E2 (PGE2) stimulates glutamate release and an increase in calcium in SVZ cells. Furthermore, they show that PGE2 is released from the choroid plexus of the lateral ventricle and the SVZ. They conclude that locally released PGE2 regulates glutamatergic signaling by controlling glutamate release from SVZ astrocytes, and thus the activity of glutamate receptors in neuroblasts. This finding may account in part for how ischemia and other pathological conditions that affect PGE2 signaling increase neurogenesis.

Calcium-dependent glutamate signaling has been shown to regulate ensheathment of Purkinje cell dendritic spines by Bergmann glia (BG) in the cerebellum. The ensheathment begins near the onset of synaptogenesis when motility of both BG processes and dendritic spines is high. Bell et al. (2010) test the hypothesis that this glial ensheathment stabilizes synapses. This was accomplished by misexpressing the AMPA receptor subunit, GluR2 in the BG, thereby causing $\mathrm{BG}$ cell process retraction as described in 2001 by lino et al.
(2001). Contrary to expectation, the data indicate that spine motility was not affected; however, the spine density increased. Thus, glial ensheathment is an important factor in closing the period of synaptogenesis. This suggests that by regulating glial ensheathment through glutamate and calcium-dependent neuron glial signaling, synaptogenesis could be promoted for therapeutic purposes.

In addition to affecting synaptic transmission, astrocytes influence many aspects of nervous system development by releasing various factors. The release of neurotransmitters, cytokines and other signaling molecules from astrocytes can affect migration, maturation, and myelin synthesis of oligodendrocytes, for example. Iacobas and Iacobas (2010) compared the gene expression profiles of precursor oligodendrocytes (Oli-neu cells) when cultured alone or in medium conditioned by astrocytes. The analysis revealed profound changes in gene expression in the Oli-neu cells, notably in myelin genes and genes involved in cytokine receptormodulated intracellular calcium signaling. Thirty-seven myelin genes were upregulated by astrocyte conditioned medium, and 14 other myelin genes were switched on. Also, genes enhancing the roles of leukemia inhibitory factor receptor and connexins 29 and 47 were increased. The enhancement in myelin genes caused by astrocyte condition medium was more profound than that seen by inducing cell differentiation using $1 \mathrm{mM}$ db-cAMP, which converts Oli-neu cells into myelin-associated glycoprotein-positive oligodendrocytes.

The final paper in this issue concerns the satellite glial cells surrounding neuronal cell bodies in trigeminal nerves, which are now known to engage in close neuronal-glial signaling. Durham and Garrett (2010) investigate the developmental changes in satellite glial cells that are likely to be involved in neuron-glial signaling and contributing to chronic pain under pathological conditions. The research shows an increase in the inward rectifying $\mathrm{K}^{+}$channel, Kir 4.1, the synaptic vesicle docking protein SNAP-25, and the calcitonin gene-related peptide by 9 days of age. The authors propose that the expression of these proteins involved in neuron-glia interactions correlate with the formation of mature functional units between these glial cells and sensory neurons in the trigeminal ganglion. The formation of these neuron-glia signaling systems is likely to be important in understanding and treating chronic pain.

The discovery of neurotransmitter release from glia adds a new chapter to the history of neurotransmission. (For a historical review of neurotransmission concepts from the classical Greek period to the discovery of nitric oxide signaling, see the review by Lopez-Munoz and Alamo (2009).) The vigorous and more rigorous experimental analysis of the poorly understood cellular mechanisms for calcium-dependent release of neurotransmitter from astrocytes and the effects on synaptic transmission that are represented by recent studies will contribute to a far better understanding the cellular basis of nervous system function.

\section{REFERENCES}

Achour S.B., Pont-Lezica L., Bechade C. and Pascual O. (2010) Is axtrocyte calcium signaling relevant for synaptic plasticity? Neuron Glia Biology 6, 147-155. 
Agulhon C., Fiacco T.A. and McCarthy K.D. (2010) Hippocampal short- and long-term plasticity are not modulated by astrocyte $\mathrm{Ca}^{2+}$ signaling. Science 327, 1250-1254.

Baltan S. (2009) Ischemic injury to white matter: an age-dependent process. Neuroscientist 15, 126-133.

Bell J.J.L., Lordkipanidze T., Cobb N. and Dunaevsky A. (2010) Bergmann glial ensheathment of dendritic spines regulates synapse number without affecting spine motility. Neuron Glia Biology 6, 193-200.

Dale H.H. (1914) The action of certain esters and ethers of choline, and their relation to muscarine. Journal of Pharmacology 6, 147-190.

Dave K.A., Platel J.-C., Huang F., Tian D., Stamboulian-Platel S. and Bordey A. (2010) Prostaglandin E2 induces glutamate release from subventricular zone astrocytres. Neuron Glia Biology 6, 201-207.

De Robertis E.D.P. and Bennett H.S. (1955) Some features of the submicroscopic morphology of the synapses in frog and earthworm. Journal of Biophysics Biochemistry and Cytology 1, 47-55.

Durham P.L. and Garrett F.G. (2010) Development of functional units within trigeminal ganglia correlates with increased expression of proteins involved in neuron-glia interactions. Neuron Glia Biology 6, $171-181$.

Fiacco T.A. et al. (2007) Selective stimulation of astrocyte calcium in situ does not affect neuronal excitatory synaptic activity. Neuron 54, 611.

Fields R.D. and Stevens-Graham B. (2002) New insights into neuronglia communication. Science 298, 556-562.

Fields R.D. and Ni Y. (2010) Nonsynaptic communication through ATP release from volume-activated anion channels in axons. Science Signaling 3, ra73.

Henneberger C. and Rusakov D.A. (2010) Synaptic plasticity and $\mathrm{Ca}^{2+}$ signalling in astrocytes. Neuron Glia Biology 6, 141-146.
Iacobas S. and Iacobas D.A. (2010) Astrocyte proximity modulates the myelination gene fabric of oligodendrocytes. Neuron Glia Biology 6, 157-169.

Iino M., Goto K., Kakegawa W., Okado H., Sudo M., Ishiuchi S. et al. (2001) Glia-synapse interaction through $\mathrm{Ca}^{2+}$-permeable AMPA receptors in Bergmann glia. Science 292, 926-929.

Katz B. and Miledi R. (1965) The effect of calcium on acetylcholine release from motor nerve terminals. Proceedings of the Royal Society of London, Series B, Biological Science 161, 496-503.

Loewi O. (1921) Über humorale Übertragbarkeit der Herznervenwirkung. Pflügers Archives 189, 239-242.

Lopez-Munoz F. and Alamo C. (2009) Historical evolution of the neurotransmission concept. Journal of Neural Transmission 116, 515-533.

Palade G.E. and Palay S.L. (1954) Electron microscope observations of interneuronal and neuromuscular synapses. Anatomical Record 118, 335-336.

Parpura V. and Haydon P.G. (2009) Astrocytes in (Patho)Physiology of the Nervous System. Springer, New York.

Shigetomi E., Kracun S. and Khakh B.S. (2010) Monitoring astrocyte calcium microdomains with improved membrane targeted GCaMP receptors. Neuron Glia Biology 6, 183-191.

Stern P.R. (2010) Reexamining glial function. Science Signaling 3, ec76.

Wiedemann C. (2010) Neuron-glia interactions: do they or don't they? Nature Reviews Neuroscience 11, 295.

Zhang Z., Chen G., Zhou W., Song A., Xu T., Luo Q. et al. (2007) Regulated ATP release from astrocytes through lysosome exocytosis. Nature Cell Biology 9, 945-953. 